\title{
Peritoneal GATA6 + macrophages function as a portal for Staphylococcus aureus dissemination
}

\author{
Selina K. Jorch, ${ }^{1}$ Bas G.J. Surewaard, ${ }^{1}$ Mokarram Hossain, ${ }^{1}$ Moritz Peiseler, ${ }^{1}$ Carsten Deppermann, ${ }^{1}$ Jennifer Deng, ${ }^{1}$ \\ Ania Bogoslowski, ${ }^{1}$ Fardau van der Wal, ${ }^{1}$ Abdelwahab Omri, ${ }^{2}$ Michael J. Hickey, ${ }^{3}$ and Paul Kubes ${ }^{1}$ \\ 'Department of Physiology and Pharmacology, Immunology Research Group, and Snyder Institute for Chronic Diseases, Cumming School of Medicine, University of Calgary, Calgary, Alberta, Canada. \\ ${ }^{2}$ Department of Chemistry and Biochemistry, Laurentian University, Sudbury, Ontario, Canada. ${ }^{3}$ Centre for Inflammatory Diseases, Monash University Department of Medicine, Monash Medical Centre, \\ Melbourne, Victoria, Australia.
}

\begin{abstract}
Essentially all Staphylococcus aureus (S. aureus) bacteria that gain access to the circulation are plucked out of the bloodstream by the intravascular macrophages of the liver - the Kupffer cells. It is also thought that these bacteria are disseminated via the bloodstream to other organs. Our data show that $\mathrm{S}$. aureus inside Kupffer cells grew and escaped across the mesothelium into the peritoneal cavity and immediately infected GATA-binding factor 6-positive (GATA6+) peritoneal cavity macrophages. These macrophages provided a haven for $\mathrm{S}$. aureus, thereby delaying the neutrophilic response in the peritoneum by $\mathbf{4 8}$ hours and allowing dissemination to various peritoneal and retroperitoneal organs including the kidneys. In mice deficient in GATA6+ peritoneal macrophages, neutrophils infiltrated more robustly and reduced S. aureus dissemination. Antibiotics administered i.v. did not prevent dissemination into the peritoneum or to the kidneys, whereas peritoneal administration of vancomycin (particularly liposomal vancomycin with optimized intracellular penetrance capacity) reduced kidney infection and mortality, even when administered 24 hours after infection. These data indicate that CATA6+ macrophages within the peritoneal cavity are a conduit of dissemination for i.v. S. aureus, and changing the route of antibiotic delivery could provide a more effective treatment for patients with peritonitis-associated bacterial sepsis.
\end{abstract}

\section{Introduction}

In humans, Staphylococcus aureus (S. aureus) bacteremia is the most common serious bacterial blood infection globally. In the United Kingdom alone, approximately 12,500 cases each year are reported with an associated $30 \%$ mortality rate (1). In a recent survey of almost 60 hospitals in the United States, $S$. aureus accounted for $23 \%$ of all infections (1). Skin and soft tissue lesions are the most common route of entry, and in a significant number of these patients, dissemination into the bloodstream leads to organ dysfunction and sepsis (2). Furthermore, with the increase in surgical interventions in hospitals and a greater number of immunosuppressed patients with chronic diseases, as well as colonization by S. aureus of indwelling catheters, prosthetic heart valves, and other foreign prosthetic devices, the number of bacteremia cases is growing each year (3). Methicillin-resistant $S$. aureus (MRSA) further complicates treatment, as there are few antibiotics available to treat this bacterium, and vancomycin is one of the last options. (2). Interestingly, the serum levels of vancomycin (and other less commonly used antibiotics) that can be achieved without causing kidney toxicity are insufficient to achieve adequate intracellular penetration and eradication of this pathogen (4). Although $S$. aureus is not thought to be an

Conflict of interest: The authors have declared that no conflict of interest exists. Copyright: ( 2019, American Society for Clinical Investigation.

Submitted: January 30, 2019; Accepted: August 1, 2019; Published: September 23, 2019. Reference information: / Clin Invest. 2019;129(11):4643-4656.

https://doi.org/10.1172/JCI127286. intracellular organism, patients are often required to receive i.v. vancomycin for weeks (if not months), in contrast to the effective staphylococcal killing observed for this antibiotic in vitro. This, and the observation that clinical infection relapse after antibiotic use is not uncommon, suggest that bacteria may hide inside cells as an evasion mechanism that facilitates their resistance to the immune response and antibiotics (5).

Despite the understanding since the early 1900 s that $S$. aureus blood infections are primarily sequestered in the liver, a reservoir for $S$. aureus that could serve as a springboard for later dissemination remained to be identified. In 1956, a seminal study by Rogers (6) showed that when blood was collected entering and leaving the visceral organs, more than $90 \%$ of the sequestration of $S$. aureus occurred via the liver within the first 10 minutes. There was always a small percentage of bacteria, however, that remained in the bloodstream and were not taken up by the liver. This was not due to saturation of clearance mechanisms within the liver, because a second large dose of bacteria was again removed with the same efficiency by the liver. This small number of bacteria were not removed from blood, because they were localized to neutrophils. In fact, 1 in every 2000 to 4000 neutrophils in the blood were infected by the bacterium. These neutrophils rather than the liver were predicted (despite the absence of experimental evidence) to be responsible for the large wave of dissemination seen 24 hours after infection. Since then, others have shown that live $S$. aureus could survive in both mouse and human neutrophils and proposed the "Trojan Horse" theory of dissemination by these immune cells (7). Transfer of $S$. aureus-infected neutrophils into the peri- 
toneum of a mouse could cause infection of the recipient mouse (8). Although these experiments indirectly support the view of $S$. aureus dissemination via the bloodstream as well as the dogma that neutrophils in the bloodstream disseminate bacteria to other organs, direct evidence, including visualization of dissemination, has not to our knowledge been provided. Moreover, S. aureus has been shown to thrive and survive in macrophages, endothelium, and other cells (9-12) making these cells as likely to be the source of dissemination. In fact, when livers from patients infected with MRSA were transplanted into recipients and whole-genome sequencing was performed, transmission of the donor MRSA strain was detected in the organ recipient (13), clearly demonstrating that a cell in the liver could also function as an MRSA reservoir in humans for subsequent dissemination.

Recently, dual-channel spinning-disk microscopy allowed for the imaging of the very rapid sequestration of $S$. aureus in blood demonstrating that it was actually the immobilized macrophages that reside in the vasculature of the liver (Kupffer cells) and not the intravascular neutrophils that sequestered more than $90 \%$ of all blood-borne bacteria $(10,14)$. Moreover, in vivo imaging revealed that some of these $S$. aureus bacteria subsequently replicated and escaped the macrophages through lysis $(10,14)$ in the first 24 hours of infection (phase 1). While the fate of these bacteria was never explored, they did not remain in the liver, as there was a marked reduction of hepatic bacteria over the first 24 hours after infection. In the second phase ( $>24$ hours after infection), bacteria began to increase in various other organs including but not limited to the kidneys. Because of the assumption that these later infections are due to dissemination via the blood, i.v. vancomycin is administered rather aggressively, often with very limited success in the short term and partial success if given for weeks (15).

To better understand the dissemination from the liver to other organs between phase 1 and phase 2 of $S$. aureus infection, we used imaging to track $S$. aureus as they left the liver, and we used the kidneys as a distal target organ. We chose to examine kidneys for dissemination studies because in mice, they typify a peritoneal organ (no retroperitoneum in mice), and we can systematically visualize allkidneycompartments.VeryfewfreeS.aureusorS.aureus-bearing neutrophils were detected in the glomerular and interstitial microvasculature of the kidney over the first or second phase of infection. By contrast, imaging revealed a different portal of infection: from the liver Kupffer cells, across the mesothelium, and into the peritoneal cavity where the bacteria took refuge in GATA-binding factor 6-positive $\left(\mathrm{GATA}^{+}\right)$peritoneal macrophages and were uncontested by neutrophils that failed to be recruited. $S$. aureus overwhelmed GATA6 ${ }^{+}$peritoneal macrophages to infect the surface of other peritoneal cavity organs including the kidneys. Consistent with this observation, i.p. administration of antibiotics that penetrated the macrophages was far superior to i.v. administration of vancomycin, prevented peritoneal organ infections, and greatly enhanced host survival.

\section{Results}

Bloodstream S. aureus infection expands from the liver into the peritoneal cavity. To induce bacteremia in our mouse model, we injected i.v. $5 \times 10^{7} \mathrm{~S}$. aureus (Newman or USA300 strain). Previous work demonstrated that the majority of bacteria are captured within the liver (10). Here, intravital microscopy of the liver vasculature (Figure 1A) revealed that GFP S. aureus (green) was almost exclusively captured by $\mathrm{F} 4 / 80^{+}$Kupffer cells (purple) and by only a minority of Ly6G neutrophils (red) within 5 minutes and phagocytosed after 20 minutes (Supplemental Video 1), as previously reported (10). Although the majority of the bacteria were killed, a small percentage (approximately 10\%) of staphylococci grew inside Kupffer cells (green dots becoming much larger after 8 hours vs. 5 minutes in Figure 1A) and formed intracellular microcolonies, as shown using higher magnification at 8 hours after infection (Figure 1B). We previously reported using 3D reconstruction and transparency of the Kupffer cells that bacteria were growing inside and not on the outside of the macrophage, demonstrating that they had been phagocytosed (10). Here, we confirmed these observations (data not shown). Over the first 24 hours, we observed a decrease of hepatic bacteria within the Kupffer cells, leaving small necrotic lesions devoid of bacteria and also loss of the podoplanin-positive mesothelium (blue) that covers the liver surface (Figure 1C). Isolation of Kupffer cells 30 minutes after infection revealed that some Kupffer cells harboring bacteria (yellow arrow) were able to prevent growth and eradicate the GFP signal over 6 hours. Other Kupffer cells (red arrow) were unable to eradicate the $S$. aureus, and the bacteria grew rapidly and ruptured the macrophage, spilling into the extracellular fluid in this cell culture system (Figure 1D and Supplemental Video 2). In vitro, about $60 \%$ of Kupffer cells eradicated bacteria (Figure 1E). Isolating the entire liver in situ 30 minutes after infection revealed that the bacteria overgrow the liver as we observed in vivo moving across the mesothelium (Supplemental Figure 1; supplemental material available online with this article; https://doi.org/10.1172/JCI127286DS1).

To determine the destination of bacteria released from the liver in vivo, a systematic assessment of the bacterial load in every organ over time was performed after i.v. injection of $5 \times 10^{7} \mathrm{~S}$. aureus. Consistent with intravital imaging, over the first 18 hours of infection, the liver harbored the majority of bacteria (Figure $1 F)$. An order of magnitude less were found in spleen and a further order of magnitude less in lung (data not shown). The profound sequestration of bacteria by liver resulted in only approximately 10,000 bacteria $\mathrm{mL}$ in the blood within 30 minutes, which further dropped to nearly sterile conditions at 18 hours (Figure 1F). Similar levels of $S$. aureus were seen in the kidney hovering at 10,000 bacteria/organ, and this value remained almost unchanged for 18 hours of infection suggesting only a tiny amount of bacteria and very little bacterial growth in this organ. In fact, the compartment with the most obvious increase in bacterial load was the peritoneal cavity. There were no bacteria detected in the peritoneal cavity over the first 12 hours, suggesting that $S$. aureus could not enter the peritoneum from the initial large bolus of bacteria injected into the bloodstream. After 24 hours, however, there was a significant and continuous rise in bacteria within the peritoneal cavity over the next 60 hours with bacteria persisting in this environment for at least 72 hours. At the beginning of phase 2, there was a small reemergence of bacteria in blood (back to 10,000 bacteria/ $\mathrm{mL}$ ) and a marked increase of infection in the kidney, of more than 1 million bacteria/kidney. By 72 hours after infection, this community-acquired $S$. aureus strain that has tropism for kidneys (16) was elevated in both the peritoneal cavity and kidneys (Fig- 
A

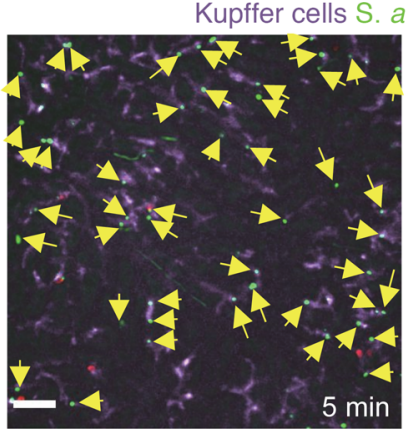

B Kupfer cells S. aureus

C

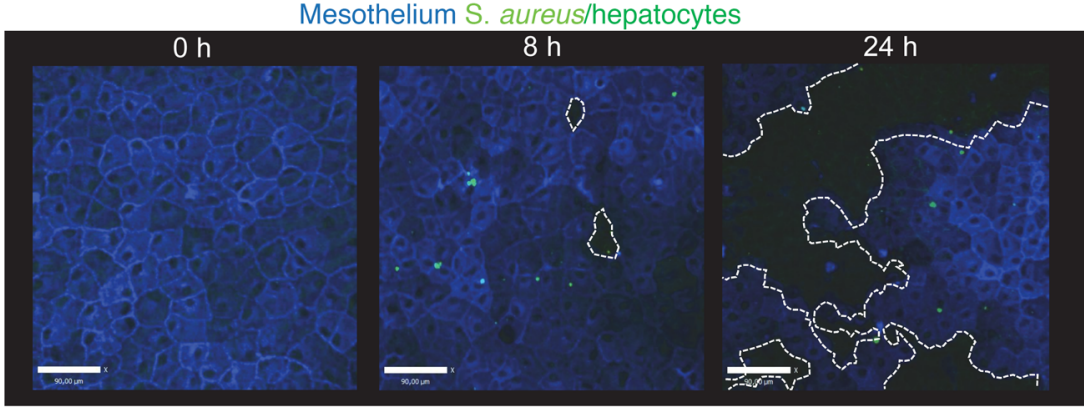

D
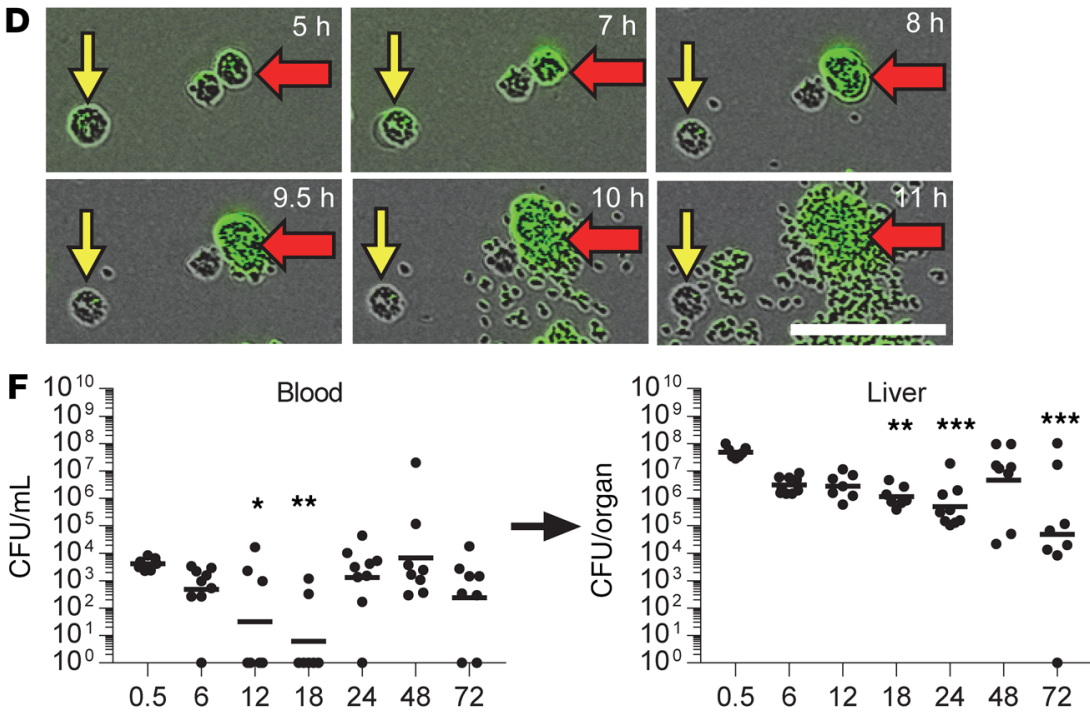

E
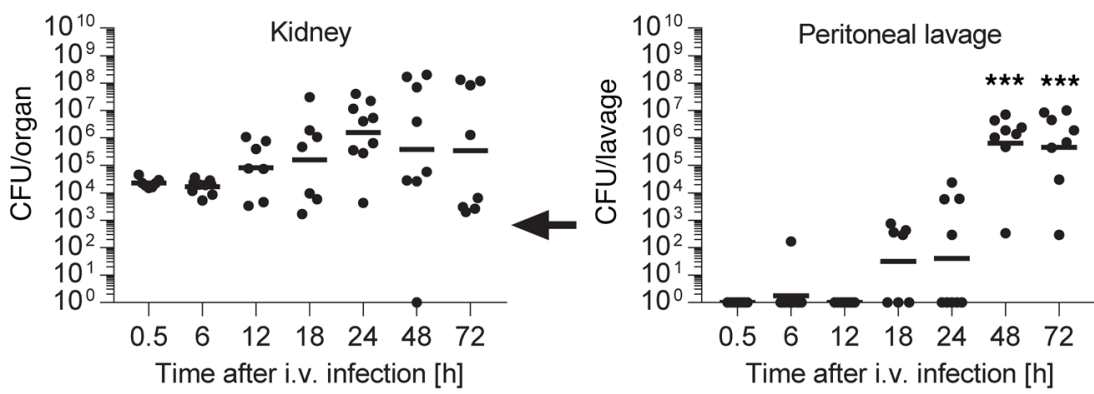

Figure 1. S. aureus escapes from liver Kupffer cells and grows into the peritoneal cavity. (A) SD-IVM images of GFP S. aureus (green) catching by Kupffer cells (F4/80; purple) 5 minutes (left; see also Supplemental Video 1) or 8 hours (right) after i.v. infection. Neutrophils are labelled in red (Ly6C). Scale bars: 50 $\mu \mathrm{m}$. (B) Enlarged view of a Kupffer cell (F4/80; purple) 8 hours after infection with growing GFP S. aureus (green) inside. Scale bar: $10 \mu \mathrm{m}$. (C) Representative images of SD-IVM of liver at indicated time points after i.v. infection. Mesothelium in blue (podoplanin-EF660), GFP S. aureus in bright green, and hepatocytes in dark green autofluorescence. Scale bar: $90 \mu \mathrm{m}$ ( $n=3$ per time point). (D and E) Kupffer cells were isolated from mice infected with GFP S. aureus for 30 minutes, cultured, and imaged over 11 hours ex vivo. (D) Representative images of time-lapse videos (Supplemental Video 2). Scale bar is $60 \mu \mathrm{m}$. (E) Analysis of time-lapse videos, 4 FOV per mouse; shown is the mean \pm SEM ( $n=6$ from 4 independent experiments). (F) Mice were infected i.v. with $5 \times 10^{7}$ CFU S. aureus. Blood was collected, peritoneal lavage was performed, and organs were harvested at the indicated time points from which CFU were determined. Shown is the geometric mean ( $n=7-9$ from 3 independent experiments), Kruskal-Wallis with Dunn's post test; ${ }^{*} P<0.05,{ }^{* *} P<0.01$, and ${ }^{* * *} P<0.001$ compared with the 0.5 -hour time point for each organ. 
A

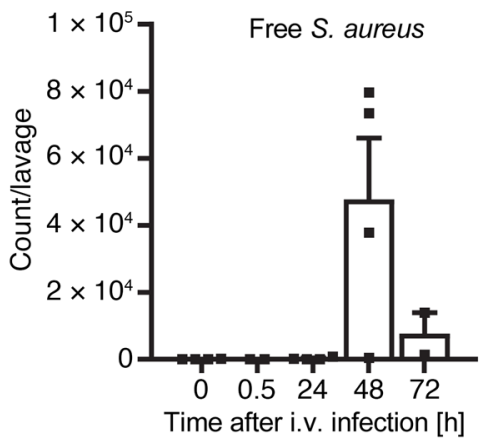

B

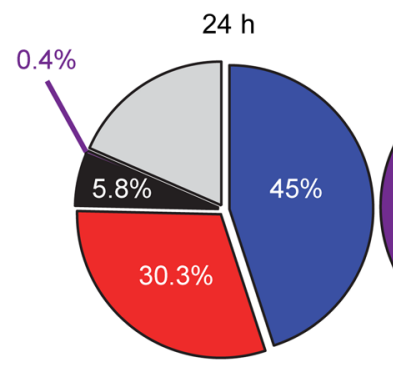

S. aureus phagocytosed by cells

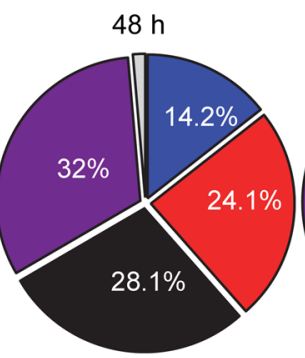

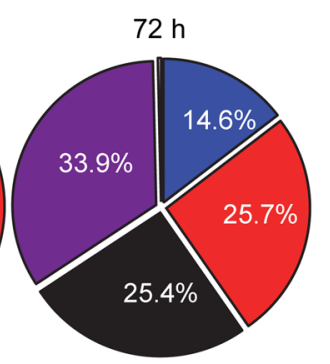

LPMs

SPMs

- Monocytes

- Neutrophils

Other CD45+ cells

C
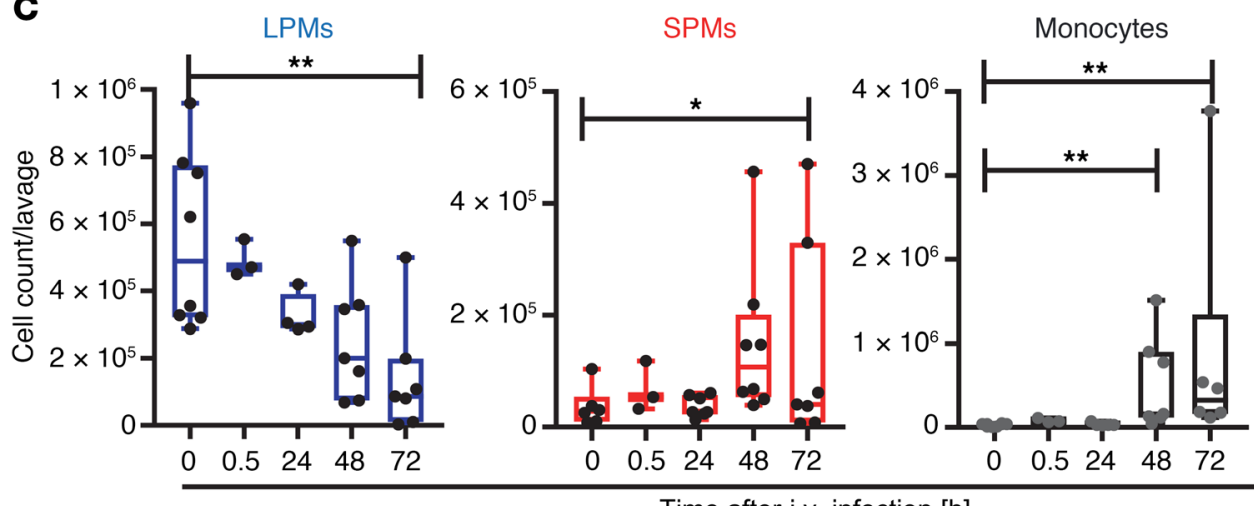

Neutrophils

Time after i.v. infection [h]

D

Peritoneal neutrophils
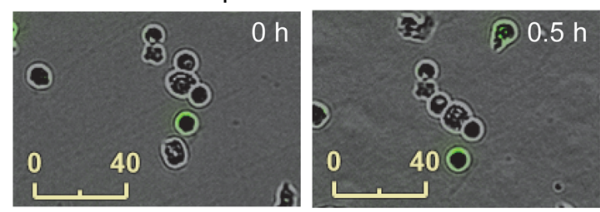

Peritoneal macrophages
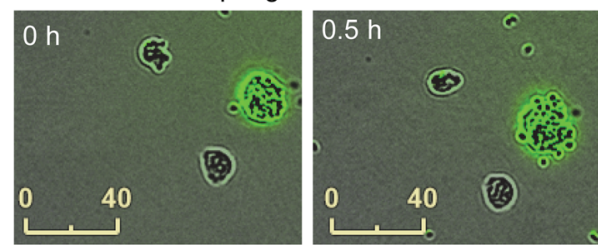
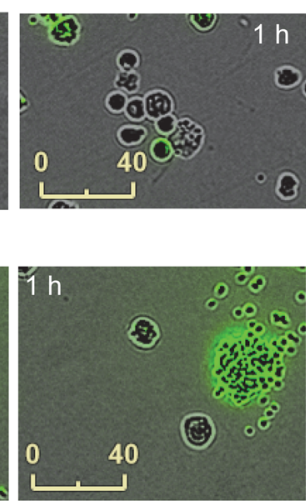
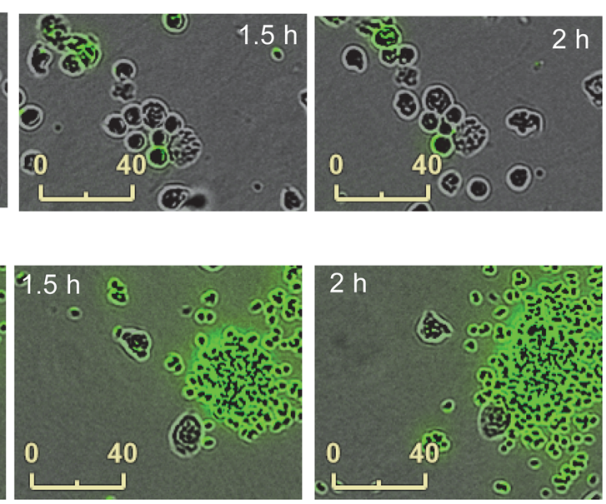

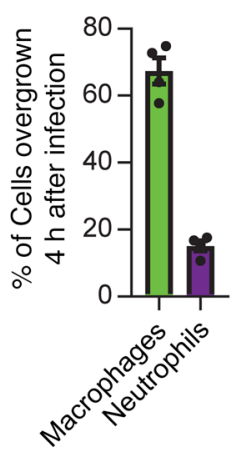

Figure 2. S. aureus infects macrophages inside the peritoneal cavity. (A-C) Flow cytometry analyses of peritoneal lavage at indicated time points after infection. The LPM and SPM gating strategy is shown in Supplemental Figure 3. (A) Total counts of free GFP S. aureus, mean \pm SEM ( $n=2-4$ from 2 independent experiments). (B) S. aureus cell localization inside the peritoneal cavity, mean percentage ( $n=6$ from 3 independent experiments). (C) Quantitative analyses for total cell count of LPMs, SPMs, monocytes, and neutrophils over infection time. Boxes extend from the 25th to 75th percentiles, whiskers show minimum to maximum, and lines indicate the median. $n=3-8$ from at least 2 independent experiments, Kruskal-Wallis with Dunn's post test, ${ }^{*} P$ $<0.05$ and ${ }^{* *} P<0.01$. (D and E) Peritoneal cells were harvested 46 hours after GFP S. aureus bloodstream infection, and F4/80+ macrophages or Ly6G+ neutrophils were isolated, cultured, and imaged ex vivo. (D) Representative images of time-lapse videos are shown (Supplemental Video 3). (E) Analysis of time-lapse videos, 4 FOV per mouse ( $n=4$ from 2 independent experiments).

ure $1 \mathrm{~F}$ ), more so than in nonperitoneal organs such as lungs (data not shown). In addition, we observed $S$. aureus in other peritoneal structures including the peritoneal wall and peritoneal visceral fat (Supplemental Figure 2) suggesting most peritoneal organs were infected (not just kidneys). The majority of animals succumbed to the infection at approximately 72 to 80 hours. The increased load of $S$. aureus in the kidneys could have come from the small increase in bacteria in blood, however, the remarkable increase in bacterial load in the peritoneal cavity was totally unexpected and therefore worthy of further examination.

Macrophages in the peritoneal cavity serve as an intracellular reservoir for $S$. aureus. A systematic assessment of the peritoneal cavity was performed with emphasis on the interplay between $S$. aureus and the innate immune cells found within this space. Figure 2A clearly shows that over the first 24 hours, no bacteria were free, and as such, any bacteria in the peritoneum were inside 
cells. There are 2 resident populations of peritoneal macrophages: the large GATA6 $^{+}$and small GATA6 ${ }^{-}$cells $(17,18)$. Over the first 24 hours after infection, most bacteria were phagocytosed by large peritoneal macrophages (LPMs; F4/80 ${ }^{\text {hi }}$, GATA6 ${ }^{+}$) and to a lesser extent by the less abundant small peritoneal macrophages (SPMs; F4/80 ${ }^{\text {intermed }}$, GATA6 $^{-}$, MHCII $^{\text {hi }}$ ) (Figure 2B, gating strategy in Supplemental Figure 3). There is a massive reserve of resident LPMs as complete uptake of the bacteria was accomplished by fewer than $10 \%$ of the total cavity macrophages. Interestingly, no neutrophils $\left(\mathrm{Ly}_{6 \mathrm{G}} \mathrm{G}^{+}\right.$) were recruited at this time and so none contributed to early bacterial uptake (Figure 2, B and C). Also, only a few proinflammatory monocytes (Ly6C ${ }^{\text {hi }}$ ) were recruited 24 hours after infection (Figure 2, B and C). Confocal imaging of Cytospin slides visually confirmed bacteria associated with LPM and SPM 24 hours after infection (Supplemental Figure 4). Interestingly, the numbers of LPMs dropped very significantly over the next 72 hours of infection (Figure 2C), reminiscent of the macrophage disappearance reaction (19). Surprisingly, neutrophils only started appearing in the peritoneal cavity at 48 hours and in much greater amounts at 72 hours after infection (Figure 2C). Previous work from us and others has shown that administration of $S$. aureus directly into the peritoneal cavity (usually at very high concentrations) recruits neutrophils within 4 hours (20-22). In this bacteremia model, where bacteria came into the peritoneum via the liver Kupffer cells, it was only at the 48-hour time point that free extracellular bacteria were detected (Figure 2A) and neutrophils began to be recruited (Figure 2C). Much like the neutrophils, monocytes that rapidly become SPMs were also only recruited into the peritoneal cavity at 48 hours (Figure 2C). Between 48 and 72 hours, the recruited neutrophils as well as some monocytes and SPMs, took up the majority of bacteria with LPMs making up only a minor component (14\%) (Figure 2B). At 72 hours, free bacteria within the peritoneal cavity decreased (Figure $2 \mathrm{~A} ; 72$ hours) and began to appear on the surface of various organs unperturbed by washing the peritoneum (shown later).

To better understand the fate of $S$. aureus within the neutrophils and LPMs, these cells were harvested and separated at 48 hours after infection for in vitro experiments. Figure 2D highlights the spectacular dichotomy in $S$. aureus handling by the 2 cell types; peritoneal neutrophils positive for GFP bacteria retained the pathogens intracellularly with very little increase in GFP signal. In contrast, LPMs frequently became overgrown by the bacteria within 2 hours, with the bacteria ultimately erupting out of these cells (Figure 2, D and E, and Supplemental Video 3) only to be taken up by more LPMs. This situation could very likely explain the significant drop in the number of these macrophages over the 72 hours of infection (Figure 2C). We hypothesized that $S$. aureus hijacked the LPMs within the peritoneum, limiting the neutrophilic and monocytic response and creating a portal for bacterial dissemination to other peritoneal organs.

Lack of $\mathrm{GATA}^{+}$peritoneal macrophages leads to less $S$. aureus dissemination to kidneys. GATA6 $6^{+}$is a specific transcription factor for the LPMs in hematopoietic cells and is not expressed in other macrophages including SPMs or Kupffer cells $(18,23,24)$ and is also not found in renal phagocytic cells, monocytes, neutrophils, or neutrophils that migrated into the kidney (Supplemental Figure 5). To determine the role of LPMs within the peritoneal cavity, we used Lyz2-Cre GATA6 $6^{f / f l}$ mice (GATA6-KO ${ }^{\text {mye }}$ ) that lack the majority of $\mathrm{GATA}^{+}$macrophages but have been reported to have no other phenotypic defects $(18,23,24)$. Not surprisingly, compared with littermate controls, these mice did not show any difference in bacterial burden in the liver (Figure 3A), as the Kupffer cell population is not altered in GATA6-KO ${ }^{\text {mye }}$ mice (24). In addition, the number of bacteria in the bloodstream was also near detection limits and not different between the 2 strains of mice (Figure 3B). At 48 hours after infection, however, we saw a significant 100 -fold reduction in bacteria associated with kidneys of mice that lacked GATA6 ${ }^{+}$LPMs demonstrating a role for these cells in S. aureus dissemination to peritoneal organs (Figure 3C). At early time points of 12 hours after infection, no difference in bacterial burden was seen in the kidneys of the WT and GATA6-KOmye mice (Figure $3 \mathrm{D})$, suggesting that small early kidney infection is unrelated to the peritoneal cavity or GATA6 ${ }^{+}$macrophages.

Bacterial burden in the peritoneal cavity was the same in GATA6-KO mye mice and littermate controls (Figure 3E). With the lack of LPMs in these mice, however, the majority of $S$. aureus were found in neutrophils (Figure $3 \mathrm{~F}$ ) in part due to a large increase in neutrophil numbers (Figure 3G). Clearly, the bacteria were unable to disseminate as effectively when in neutrophils instead of GATA6 ${ }^{+}$ macrophages. GATA6-KO ${ }^{\text {mye }}$ mice also appeared healthier and lost less body weight than littermate controls (Figure 3H).

Direct injection of $S$. aureus into the peritoneal cavity caused a very significant early (4 hours) increase in neutrophils (1-2 million) into the peritoneal cavity (Figure 3I) with some kidney infection at 72 hours (Figure 3J) as previously described (25). In GATA6deficient mice, the neutrophil influx was tripled (4-6 million), and less bacterial burden was noted in the kidneys 3 days after i.p. infection (Figure 3, I and J). When we measured the levels of neutrophil-recruiting chemokines, CXCL1, and CXCL2, within the peritoneal cavity, we were surprised to find very robust increases 4 hours after i.p. injection of $S$. aureus but not after the first 24 hours of i.v. injection of the bacteria (Supplemental Figure 6).

S. aureus infects the kidney primarily from the peritoneum not vasculature. The Leica Dive 2-photon intravital microscope (IVM) was used to visualize S. aureus in the microvasculature of the kidney, including that of glomeruli, which in adult mice are almost impossible to visualize with standard IVM due to their depth below the surface. We were also able to stitch images such that one could observe as many as $\mathbf{2 0}$ glomeruli simultaneously, which could then be magnified as needed and appear as small grey spheroids (Figure 4A and Supplemental Figure 7A). Despite massive sequestration of $S$. aureus in the hepatic microvasculature (Figure 1A), very few bacteria were observed adhered (stationary) in either the interstitial or glomerular microvasculature of the kidney (Supplemental Videos 4-6) in the first 30 minutes of infection. In addition, on rare occasions, we observed ingested bacteria in neutrophils attached to glomerular capillaries, but these cells then detached from the kidney vasculature and reentered the mainstream of blood (Figure 4B and Supplemental Video 5). Neutrophils were never observed to undergo prolonged retention in the kidney vasculature. There was no persistent growth of $S$. aureus in any of the many glomeruli in the countless mice we imaged. In fact, we found only 1 glomerulus infected with $S$. aureus at 72 hours after infection (Supplemental Figure 
A

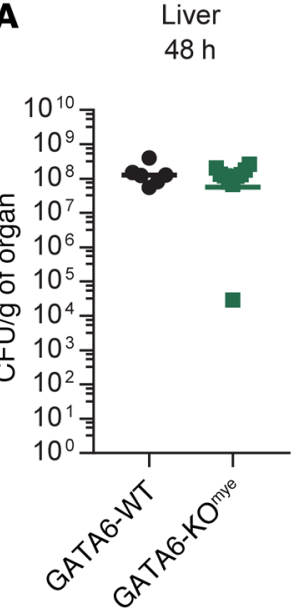

D

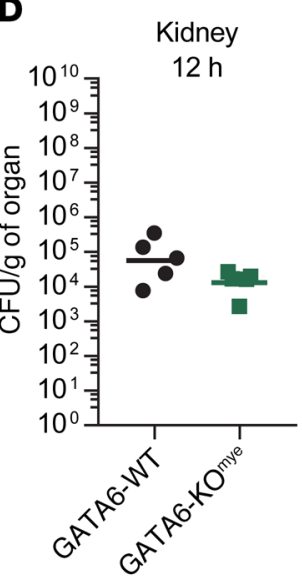

G

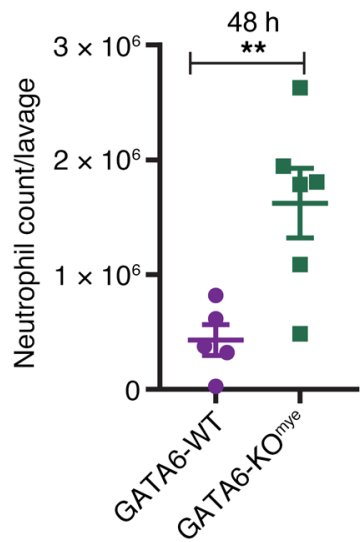

B

Blood
$48 \mathrm{~h}$

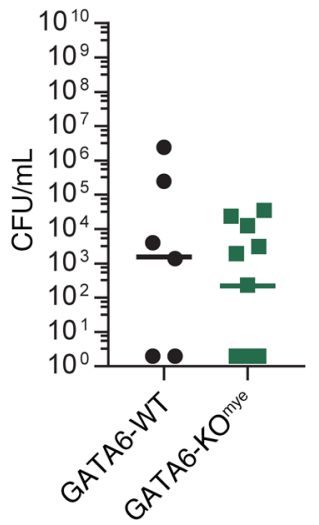

E Peritoneal lavage

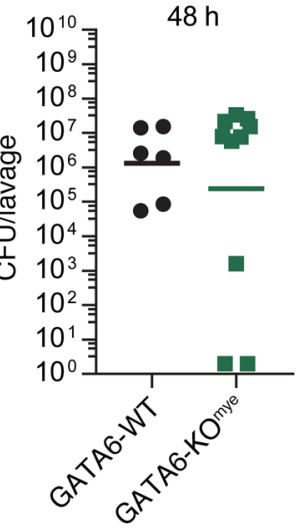

H

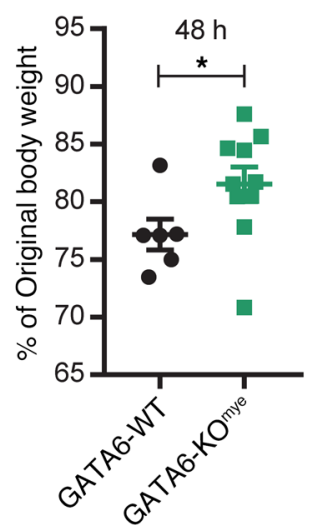

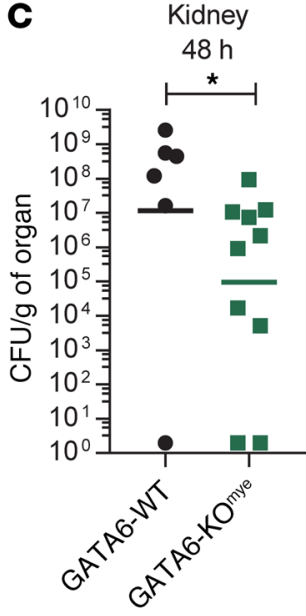

$\mathbf{F}$
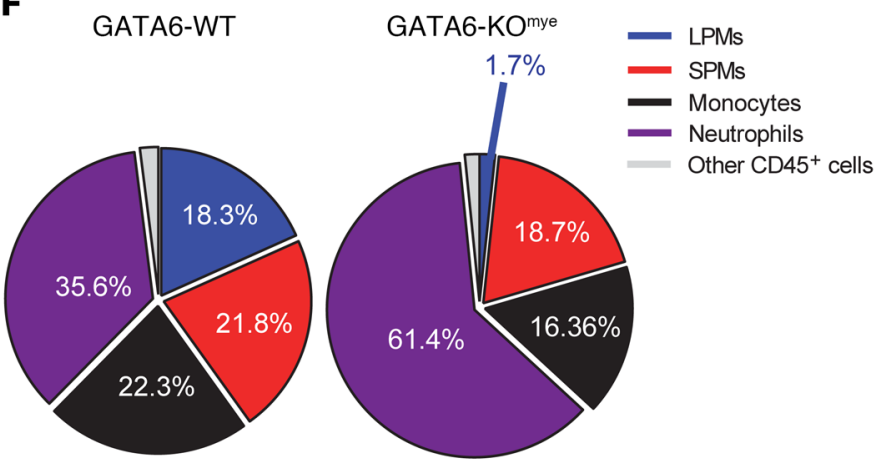

I

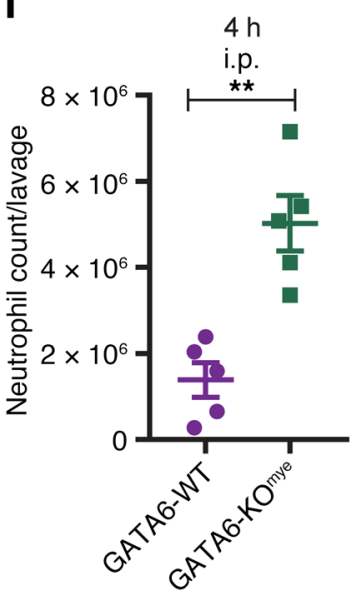

$J$

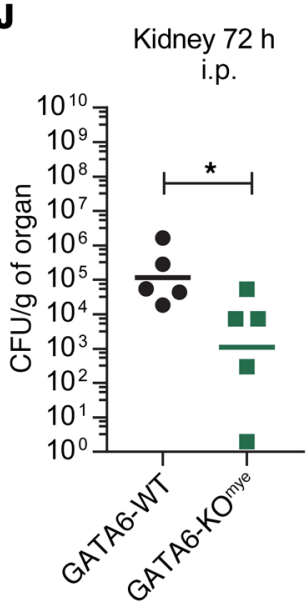

Figure 3. A lack of peritoneal macrophages results in more neutrophil recruitment and less dissemination of $\mathbf{S}$. aureus to the kidneys. CATA6-KOmye mice or littermate controls were infected i.v. (A-H) or i.p. (I and J) for indicated time points with $5 \times 10^{7}$ S. aureus Newman strain. (A-E and J) Organs were collected and the CFU determined; the geometric mean is shown. $n=5-10$ from at least 2 independent experiments, Mann-Whitney test, ${ }^{*} P<0.05$. (F) S. aureus cell localization inside the peritoneal cavity determined by flow cytometry, mean percentage. $n=5$ (WT) or 6 (GATA6-KO ${ }^{\text {mye }}$ ). (G and I) Neutrophil numbers in the peritoneal cavity assessed by flow cytometry. $n=5-6$, mean \pm SEM, unpaired $t$ test, ${ }^{* *} P<0.01$. (H) Body weight loss, $n=6-9$ from 3 independent experiments, mean $\pm \mathrm{SEM}$, unpaired $t$ test, ${ }^{*} P<0.05$.

7A). In addition, some studies suggest that CX3CR1 $1^{+}$renal phagocytes monitor the kidney microvasculature and catch Gramnegative bacteria and particulate material from the circulation $(26,27)$. In our imaging experiments, however, no CX3CR1 $1^{+}$renal phagocytes contained any bacteria in the first 30 minutes after i.v. infection (Supplemental Video 6), and flow cytometry data show that this does not change over time, never increasing above $0.5 \%$ (Supplemental Figure 7B). While the very few bacteria seen in the 
kidney using imaging are entirely in line with the very few CFU $\left(10^{4} \mathrm{CFU}\right)$ detected over the first 24 hours, the lack of neutrophil recruitment and lack of bacteria in the different compartments of the kidney were not consistent with the increased bacterial load in kidneys 24 hours onward.

Interestingly, there was no increase in adherent neutrophils within glomeruli or the interstitial microvasculature until 72 hours after infection (Supplemental Figure 8A). Neutrophils were retained in both glomeruli and the interstitial microvasculature of the kidney for approximately 2 to 3 minutes under basal conditions, and this value did not increase over time after infection (Supplemental Figure $8 \mathrm{~B}$ ) suggesting little evidence of inflammation in the renal vasculature. We also assessed renal leukocyte recruitment via flow cytometry of digested kidneys. These experiments revealed a minor increase in the total number of neutrophils in the kidney at 48 hours followed by dramatic recruitment at 72 hours after infection (Supplemental Figure 8, C and D). It is noteworthy that very few neutrophils had ingested $S$. aureus during the first 24 hours, and even at 48 and 72 hours, only $15 \%$ of recruited neutrophils were positive for GFP S. aureus (Supplemental Figure 8, E and $\mathrm{F})$, indicating poor access to the bacteria.

The blood neutrophil counts were also examined using flow cytometry to determine the number of neutrophils that had bound or ingested $S$. aureus. The number of GFP $S$. aureus-bearing neutrophils was consistently less than $0.1 \%$ of the total for the entire 72 hours after infection (Supplemental Figure 9) despite a huge increase in bacterial load associated with the kidneys. Together with the imaging showing no bacteria in the vasculature and these flow cytometry data, there was a lack of convincing support for the prevailing view that bacteria are reaching the kidney through the vasculature inside neutrophils or even as free entities.

Because the bacteria did not appear to be disseminating via the vasculature, we visualized the kidneys from the peritoneal aspect and in overview pictures, we saw increasing growth of bacteria on the renal capsule at 24 hours (not shown), 48 hours, and 72 hours (Figure 4C). Although at 24 hours the bacteria seemed to be in tiny colonies firmly adherent and sprinkled across the surface of the kidney capsule (not shown), these colonies grew noticeably at 48 hours forming biofilms with concentrated areas of intense fluorescence suggesting isolated areas of large colonies. Whereas this occurrence has not been reported in humans, it is unlikely these clumps of bacteria would be detected by ultrasound and other clinical approaches. It is noteworthy that the average bacterial load did not change significantly between 24 and 72 hours, and the bacteria seemed to be much less dispersed and in more concentrated clumps at later time points. Two-photon microscopy and the use of second-harmonic generation (denotes collagen) to delineate the location of the kidney capsule revealed bacteria attached to the surface and not below the capsule at 48 hours (Figure 4D and Supplemental Video 7), whereas at 72 hours, bacteria became visible deeper in the parenchyma (Figure 4E). By 72 hours, the infection had spread from the surface of the kidney to the interstitium but was almost never visible in glomeruli (Supplemental Figure 7A). Quantification of the total number of individual bacteria/bacterial clusters revealed that at 24 hours, all bacteria were on the renal capsule, while at 48 and 72 hours, there were increasing numbers of bacteria in the interstitium (Figure $4 \mathrm{~F}$ ).
This observation suggested that bacteria were infecting the kidney from the outer surface inward via the peritoneal cavity. Consistent with this idea, it is well known that injection of bacteria directly into the peritoneal cavity leads to abscess formation initially on the kidney surface followed by invasion into the interstitium (16, 25), a frequently used model of renal failure. We also assessed cell death in the kidney and observed very little tubular cell death at 24 hours, but this was greatly increased at 48 and 72 hours (Supplemental Figure 8, G and H). Plasma creatinine was also measured as an index of glomerular filtration rate and impaired renal function, but the data showed no correlation with local infection. There was no increase in serum creatinine at any time point except at 24 hours (Supplemental Figure 4I). The temporary early increase in creatinine is consistent with our report that $\alpha$-toxin released into the bloodstream from liver- sequestered $S$. aureus caused platelet aggregation in capillaries including kidney glomeruli leading to temporarily impaired filtration (28).

Eradication of the $S$. aureus reservoir in the peritoneal cavity with antibiotic-filled liposomes results in better survival. For bloodstream infections in humans, i.v. vancomycin, a potent antibiotic for $S$. aureus, is recommended for a minimum of 4 to 6 weeks, although success can be limited (5). In the present experiments, i.v. vancomycin administration for 1,2 , or 3 days starting 24 hours after infection (to mimic the clinical situation) had a marginal effect on the bacterial load associated with the kidney or the peritoneum at 72 hours (Figure 5A and Supplemental Figure 10A). Moreover, the number of dead tubular cells (Figure 5, B and C), weight loss (Supplemental Figure 10B), and survival (Figure 5D) were not improved. Interestingly, splitting of vancomycin dose and administering half i.v. and the other half into the peritoneal cavity reduced infection in the peritoneal cavity, reduced the number of bacteria and tubular damage in the kidneys, and significantly improved mortality rates (Figure 5, A-D). We previously reported that free vancomycin did not penetrate well into tissue macrophages (10). In contrast, vancomycin incorporated into liposomes (vancosomes), was voraciously taken up by Kupffer cells 30 minutes after infection helping to eradicate $S$. aureus inside these cells (10). However, the 30 minutes after infection protocol used in our previous study, while sufficient for proof of concept, is unlikely to be clinically relevant. Because the bacteria are released from the liver into the peritoneal cavity at approximately 8 hours, administering i.v. vancosomes at 24 hours would be too late. Therefore, we injected vancosomes i.p. at 24 hours and observed a massive uptake of these liposomes by peritoneal macrophages. It is worth noting that when liposomes were given i.p., they were able to access the vasculature where they were taken up by Kupffer cells but not by renal phagocytic cells (Supplemental Figure 11). Therefore, the vancosomes were not functioning within kidney macrophages.

The profound reduction in bacterial load in the peritoneal cavity after vancosomes lowered the bacterial load in kidneys in 50\% of mice (Figure 5A) and led to less kidney injury (Figure 5, B and C) and better survival (Figure 5D). Interestingly, some bacteria still managed to grow on the kidney capsule suggesting the vancosomes were less effective at killing extracellular bacteria. We observed better eradication of free bacteria with vancomycin and better eradication of intracellular bacteria with vancosomes (Supplemental Figure 10C). The combination of vancomycin i.v. and 
A
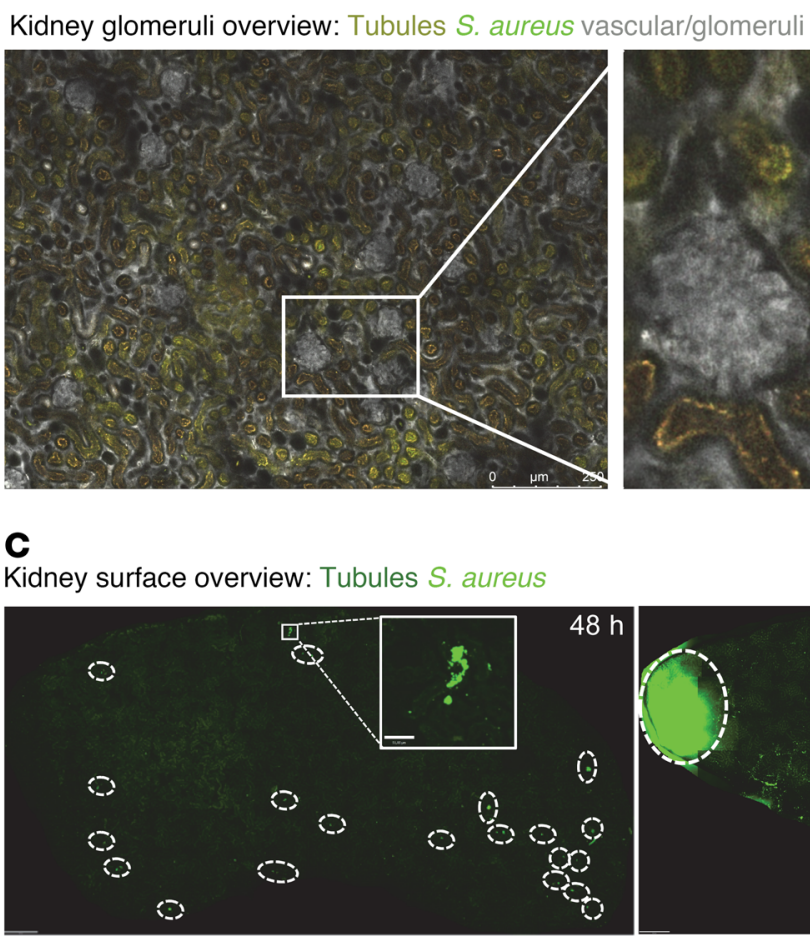

C

Kidney surface overview: Tubules S. aureus
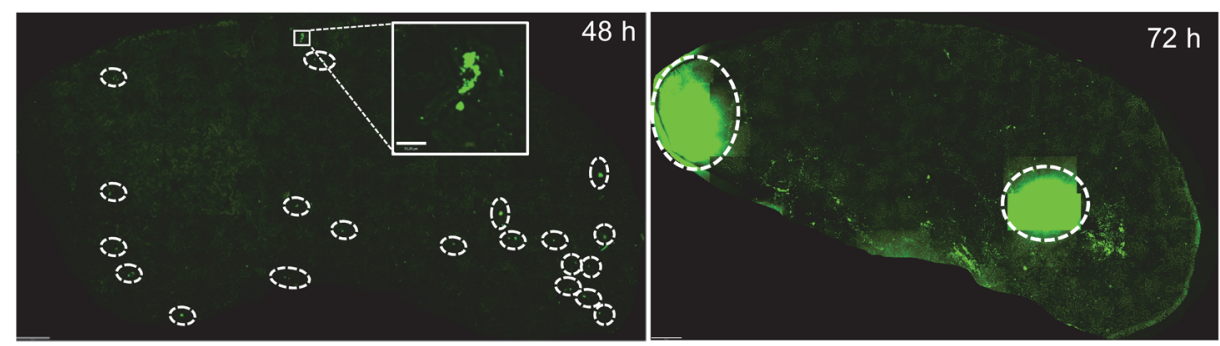

\section{D}

Collagen (SHG) S. aureus

Vascular (incl. glomeruli)

Tubules

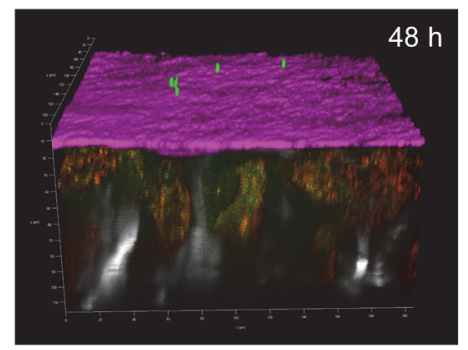

E

S. aureus

Tubules

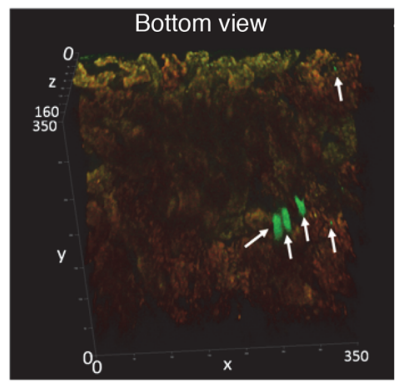

B

Glomerulus Neutrophils

S aureus-GFP inside a neutrophil
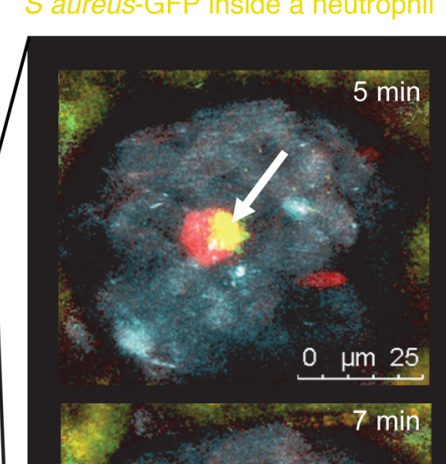
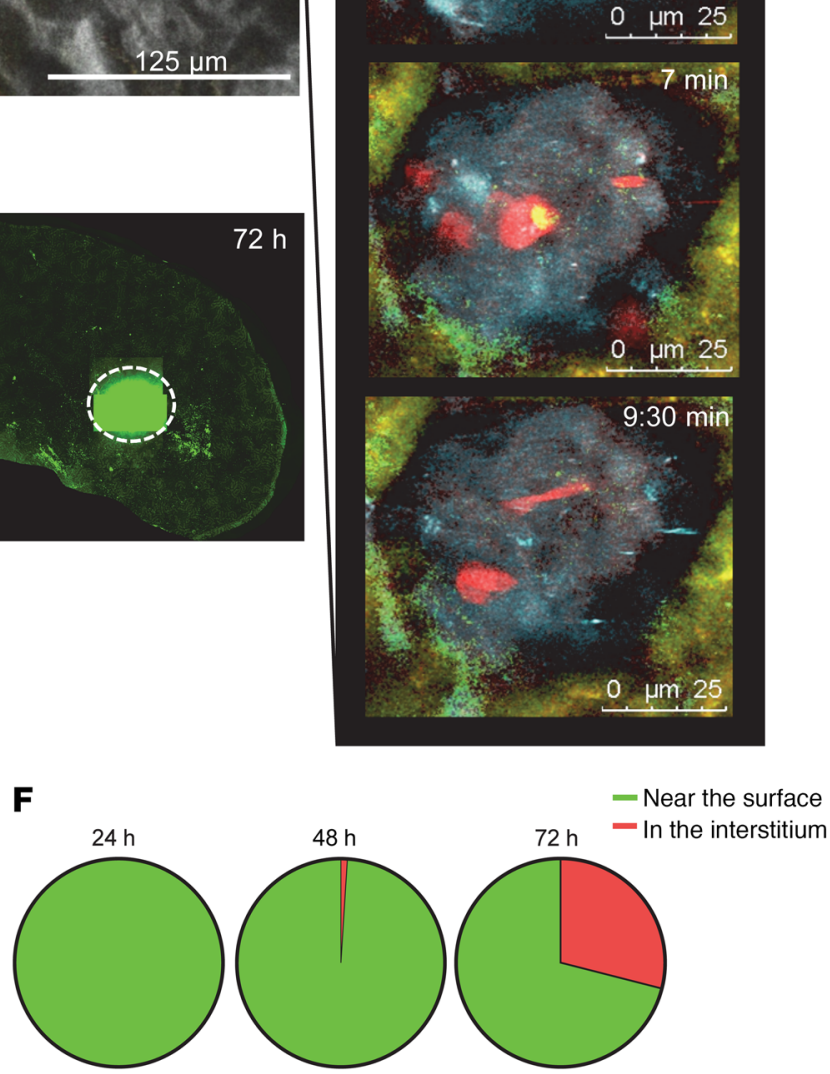

Figure 4. S. aureus infects the kidney initially on the capsule and then infiltrates into the interstitium. (A) Representative 2-photon stitched image from kidney around $150 \mu \mathrm{m}$ deep where approximately 20 glomeruli can be seen (left). Vasculature including glomeruli appear in grey (AF680-albumin), tubules appear in dark yellow autofluorescence, and S. aureus is in bright green GFP (not visible). The right panel shows 3 enlarged glomeruli. (B) Two-photon IVM images from a glomerulus (Supplemental Video 5) during the first 10 minutes of i.v. infection with $5 \times 10^{7}$ S. aureus Newman. Vasculature (glomerulus) is shown in blue (Qtracker 655), tubules are autofluorescent in all channels and appear in brown around the glomerulus, neutrophils are labelled in red (Ly6G-PE), and GFP S. aureus appears in yellow inside a neutrophil (white arrow). (C) Representative stitched images from kidney surfaces at indicated time points after infection. Scale bar: $500 \mu \mathrm{m}$. S. aureus (bright green) is circled in white and an enlarged view of bacteria on the surface at 48 hours is shown. (D) 3D representation FOV of a kidney 48 hours after infection with GFP S. aureus (bright green) on the capsule (purple collagen/second harmonic generation [SHG]) and the vasculature in grey (AF680-albumin). (E) Representative FOV of a kidney 72 hours after infection, view from the bottom of the $Z$-stack. Tubules appear autofluorescent in brown, GFP S. aureus is bright green and indicated with white arrows. (F) Analysis of location of bacteria as determined using 2-photon IVM of the kidney. Six FOV per mouse were analyzed ( $n=3-4$ per time point).

vancosomes i.p., which would be the most likely choice in a clinical trial, cleared all bacteria in the peritoneal cavity in all mice, and no bacteria reached the kidneys in more than $50 \%$ of mice (Figure $5 \mathrm{~A})$. When mice were treated with just 1 injection at 24 hours, the results were moderately less effective (Supplemental Figure 10A). Most importantly, mice treated with tandem vancosomes i.p. and vancomycin i.v. increased survival by $80 \%$ at 10 days despite only a 3-day antibiotic regimen (Figure 5D). These protocols could be readily translated into clinical practice in infected patients.

\section{Discussion}

The role of neutrophils in S. aureus infections is irrefutable. Making mice neutropenic makes animals very susceptible to $S$. aureus infection (29). Moreover, neutropenia is the greatest risk factor for developing S. aureus bacteremia (30). Nevertheless, while performing their main role to eradicate $S$. aureus infection, the neutrophils may inadvertently cause dissemination of the pathogen. Numerous groups have reported that $S$. aureus can survive within neutrophils and potentially disseminate pathogens via the blood- 
stream $(6,8,31,32)$. Certainly, we also saw a low amount of bacteria $(\sim 10,000)$ in the kidney 0.5 hours after infection, consistent with the view that a small percentage of infected neutrophils could potentially transmit some bacteria to the kidneys via intravascular dissemination. The very few bacteria that were detectable by imaging the kidney likely equate to the few CFU observed, but a limitation of imaging may be that the $S$. aureus are also located in areas not penetrated by 2-photon microscopy and some infection could be missed. However, neutrophils are not the only cells that become infected by $S$. aureus. In our study, when bacteria first entered the bloodstream, more than $90 \%$ of them were taken up primarily by Kupffer cells, which could also help to disseminate bacteria. $S$. aureus escaped Kupffer cells, entered into the peritoneal cavity, and used an evasion mechanism, hiding inside peritoneal macrophages thereby being protected from neutrophils. In fact, neutrophils did not appear within the peritoneal cavity until 48 hours after infection when bacteria had reached peak levels within this compartment, including growing on the kidney capsule.

Therefore, the majority of dissemination appeared to occur via the peritoneal cavity. Our study demonstrates that in mice, $S$. aureus uses tissue macrophages as a dissemination portal, first growing inside Kupffer cells, then outgrowing these cells and rupturing into the peritoneal cavity where it thrives inside the GATA6 ${ }^{+}$ peritoneal cavity macrophages prior to forming colonies on the surface of kidneys. While in humans, the kidneys are found in the retroperitoneum, which may make it harder for $S$. aureus to reach this site, it was not just the kidneys that were infected. Examining the peritoneal wall and visceral fat also showed bacterial growth, clearly suggesting dissemination into numerous organs within the peritoneal cavity. In humans, liver abscesses often occur and have to be drained, as otherwise, they would rupture filling the peritoneal cavity with bacteria, leading to bacterial dissemination (33, 34). However, smaller abscesses as those noted herein in mice would almost certainly go unnoticed in humans and could serve a similar dissemination route.

Recent work has revealed that the peritoneum harbors 2 types of macrophages. The smaller but recruitable population are the monocyte-derived small peritoneal macrophages that are GATA6 and important in inflammatory responses and infections $(17,35)$. The larger population is a resident pool of yolk sac-derived large peritoneal macrophages that are $\mathrm{GATA}^{+}$and have been reported to disappear during infections $(19,36)$. These macrophages rapidly convert to a repair phenotype and are critical for repairing visceral organs (24). To date, they have not been thought to play a major role in infections. Our data suggest, however, that $S$. aureus actually subverts this most abundant population of resident macrophages. These macrophages rapidly phagocytose $S$. aureus after its entry into the peritoneal cavity, although they are not capable of killing the pathogen. Instead, we observed a very profound growth of $S$. aureus within the peritoneal macrophages, which ultimately led to overgrowth of these cells, cell lysis, bacterial release, and reuptake by more macrophages until the GATA $6^{+}$macrophage disappeared and the bacteria attached to the surface of kidneys where they formed colonies. It is interesting that once $S$. aureus was attached to the surface of the kidneys, none of the immune cells was very effective at eradicating the infection, raising the possibility these large clumps of bacteria have now formed impenetrable biofilms.
Under these conditions, the bacterial load did not increase over the infection period also consistent with a phenotypic change of the $S$. aureus from planktonic to biofilm.

Although previous work has shown that there are macrophages in skin, lung, and liver that are important in rapidly recruiting neutrophils $(10,37,38)$, this was not the case for the GATA6 ${ }^{+}$ macrophages in our systemic infection model. Despite macrophages phagocytosing the $S$. aureus, no neutrophils were observed for 48 hours in the peritoneal cavity, allowing the pathogen to disseminate. One possibility is that the $\mathrm{GATA}^{+}$macrophages are unable to recruit neutrophils; however, our previous studies and others have shown that these macrophages can recruit neutrophils in response to many different proinflammatory reagents and bacteria when injected i.p. $(39,40)$. The difference is that injection of huge amounts of bacteria or bacterial products into the peritoneum causes the release of neutrophil-recruiting chemokines, whereas in our intravascular model, small numbers of bacteria were entering the peritoneum from Kupffer cells and immediately taken up by GATA6 ${ }^{+}$macrophages, and no neutrophil-recruiting chemokines were detected. In addition to limited numbers of $S$. aureus entering the peritoneal cavity, it is also possible that while the bacteria were incubating inside the Kupffer cells for at least 8 hours, they could have modified their phenotype, becoming more inert and less metabolically active not dissimilar to small-colony variants that may not activate the GATA6 $6^{+}$macrophages (41). This finding is consistent with our data showing an absence of CXCL1 and CXCL2 in our model but not when large amounts of bacteria were injected directly i.p. It was only when the bacteria overgrew the peritoneal macrophages at 48 hours that neutrophils started infiltrating into the cavity. An absence of GATA6 $6^{+}$macrophages allowed neutrophils to infiltrate in greater numbers, but even under these conditions, neutrophils were significantly delayed compared with the previously reported rapid 4-hour neutrophil recruitment that occurs when $S$. aureus is injected directly into the peritoneum. This finding is consistent with $S$. aureus being mechanically and/or phenotypically cloaked in some manner after emerging from Kupffer cells. A final possibility is that when the neutrophils phagocytose $S$. aureus and are then taken up by macrophages, this could also function to dampen inflammation. Neutrophils have been show to enter all tissues (42), so they would potentially already be there, as the $S$. aureus escaped Kupffer cells and entered the peritoneum. Neutrophil phagocytosis by macrophages is a well-known suppressor of phlogistic activity in macrophages (42).

The standard of care following $S$. aureus detection in blood is high-dose i.v. vancomycin for 6 weeks or longer (5). Our data suggest that there are huge limitations to standard-of-care i.v. vancomycin alone including complete inability to penetrate into the very cells in which the bacteria are hiding, namely the tissue macrophages of both the liver and the peritoneal cavity. The latter may be further hampered by the fact that i.v. vancomycin administration poorly sterilizes the peritoneal compartment, a lesson learned from patients undergoing peritoneal dialysis. Simply splitting the dose of vancomycin i.v. and i.p. (total concentration being equal) starting 24 hours after infection for only 3 days, greatly improved survival of mice for 10 days (duration of experiments). Further delivery optimization by encapsulating vancomycin into liposomes (vancosomes) 
A

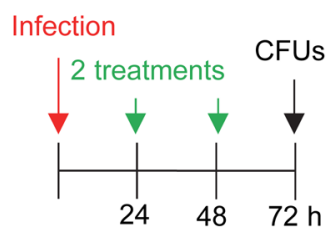

B

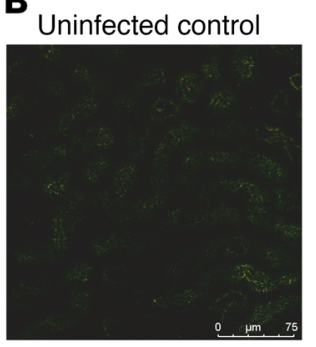

Vancomycin i.v. + i.p.
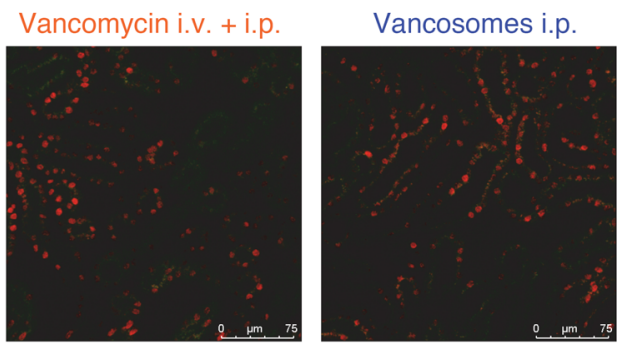

Peritoneal cavity

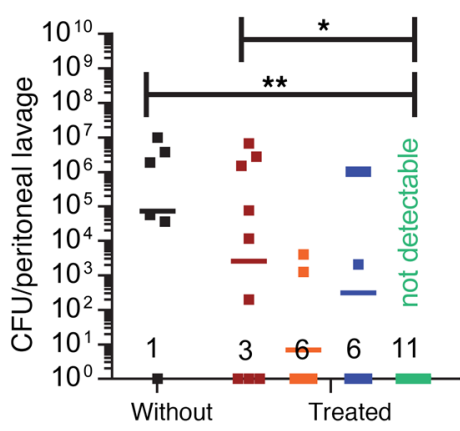

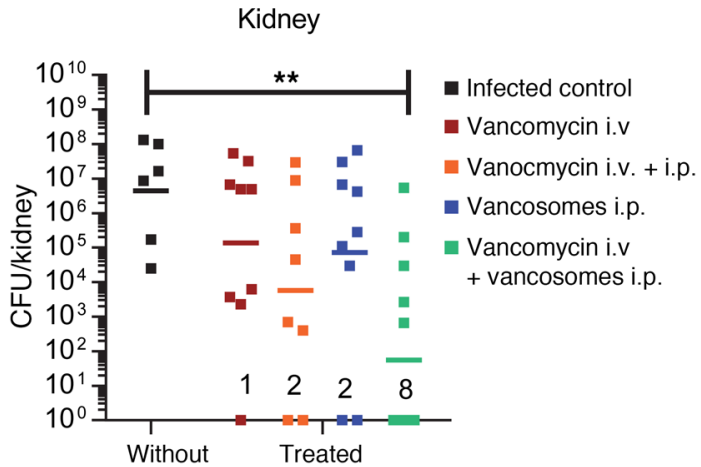

C

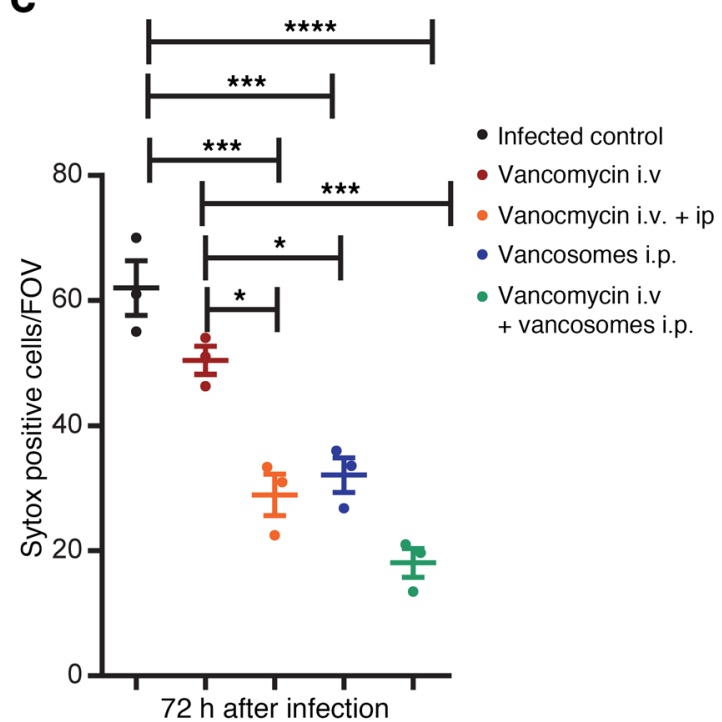

D
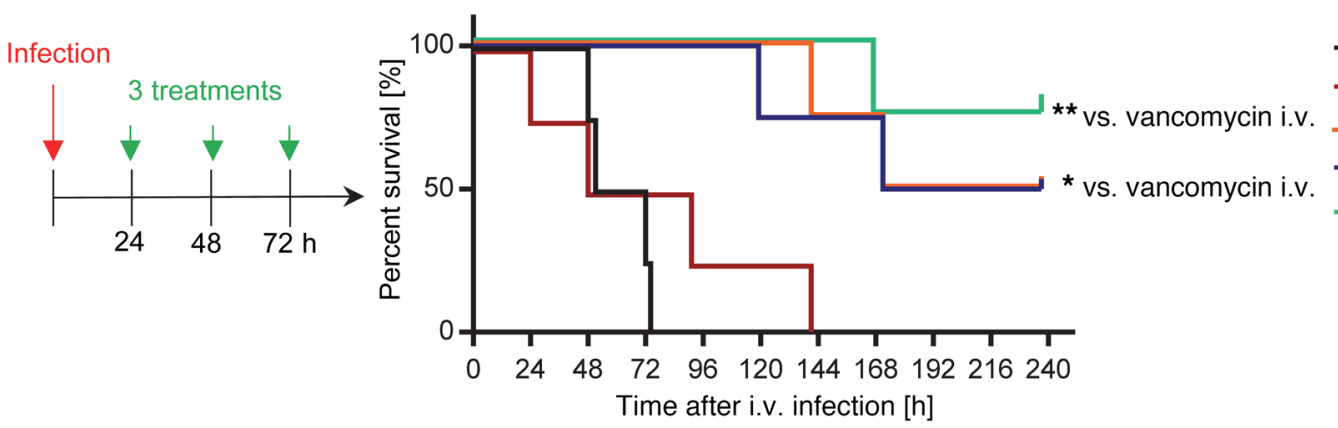

Figure 5. Intraperitoneal liposomal vancomycin (vancosomes) targets tissue resident macrophages to eradicate infections and sustain survival. Mice were infected i.v. with $5 \times 10^{7}$ S. aureus Newman and treated 2 ( 24 hours plus 48 hours, A-C) or 3 times ( 24 hours plus 48 hours plus 72 hours, D) with either vancomycin i.v (red), vancosomes i.p. (blue), a combination of vancomycin i.v. plus i.p. (orange), or vancomycin i.v. plus vancosomes i.p. (green). (A) Seventy-two hours after infection, organs were harvested, peritoneal lavage was performed, and CFU were determined ( $n=6-11$ from 3 independent experiments). Data are presented as the geometric mean; Kruskal-Wallis with Dunn's post test; ${ }^{*} P<0.05$ and ${ }^{* *} P<0.01$. (B) Representative 2-photon IVM images of kidneys 72 hours after infection with or without treatment 2 times. Dead tubular cells are stained with Sytox Orange (red), tubules appear in dark green autofluorescence. Scale bar: $75 \mu \mathrm{m}$. (C) Analysis of B. Number of Sytox Orange (dead) cells from 8 FOV per mouse, data represent the mean \pm SEM ( $n=3$, 1-way ANOVA with Bonferroni's multiple comparisons test). ${ }^{*} P<0.05,{ }^{* * *} P<0.001$, and ${ }^{* * * *} P<0.0001$. (D) Survival curve of $S$. aureus-infected and treated mice ( $n=4$ mice per group from 2 independent experiments). Log-rank test compared with vancomycin i.v., ${ }^{*} P<0.05$ and ${ }^{* *} P<0.01$.

improved drug uptake by peritoneal macrophages, the very cells that were harboring the pathogen helping to kill these bacteria.

Clearly, S. aureus has evolved to survive in tissue macrophages and these cells, found in liver, spleen, skin, peritoneum, bone, and all other tissues may function as a safe haven and conduit for fur ther dissemination (43). Bacteremia leads to rapid sequestration by Kupffer cells, which unwillingly transfer the bacteria to the peritoneal macrophages that function as a portal to other visceral organs. Moreover, our work shows that $S$. aureus has also found a way to penetrate through the mesothelium, giving the bacteria 
free access throughout the peritoneum including perhaps the retroperitoneum, which is separated by a layer of mesothelium and harbors the kidneys in humans but not mice. It remains unknown how the bacteria cross the mesothelium, but the fact that $S$. aureus causes abscesses in skin that burst through multiple layers of dermal tissue makes rupture into the peritoneum across the one cell layer of mesothelium a likely proposition. Although the peritoneum is almost certainly not the only portal for dissemination (the bacteria could re-enter the bloodstream from the peritoneum), it is one that could be targeted more effectively with the addition of antibiotic both i.v. and into the peritoneal cavity.

\section{Methods}

Animals. Five- to 8-week-old male and female C57BL/6 mice and CX3CR1 $1^{\mathrm{GFP} /+}$ mice were obtained from The Jackson Laboratory. Gata6-floxed mice were provided by Ruslan Medzhitov (Yale University, New Haven, Connecticut, USA) and bred in-house with Lyz2-Cre mice (The Jackson Laboratory). Lyz2-Cre ${ }^{+} \mathrm{Gata}^{\mathrm{fl} / f l}$ mice were subsequently bred with $\mathrm{Lyz2}^{-} \mathrm{Cre}^{-} \mathrm{Gata} 6^{\mathrm{fl} / \mathrm{ll}}$ mice to generate $\mathrm{Cre}^{+}$and $\mathrm{Cre}^{-}$ littermates. All mice were housed in a specific pathogen-free, doublebarrier unit at the University of Calgary, with access to tap water and pelleted food ad libitum.

Bacteria and culture conditions. S. aureus Newman strain or USA300 were used, when necessary transformed with pCM29 to constitutively express high levels of GFP or mCherry. Bacteria were grown in brain heart infusion at $37^{\circ} \mathrm{C}$ with shaking overnight. Chloramphenicol $(10$ $\mu \mathrm{g} / \mathrm{mL}$ ) was added for maintenance of the plasmid. Bacteria were subcultured before i.v. infections. For experiments with $\mathrm{CX} 3 \mathrm{CR} 1^{\mathrm{GFP} /+}$ mice, non-GFP bacteria were labelled with AF594. Fresh staphylococcal cultures were washed twice in saline, resuspended at $5 \times 10^{8} \mathrm{CFU}$ in $500 \mu \mathrm{L}$ carbonate-buffered saline ( $\mathrm{pH} 8.3$ ), and labeled for 30 minutes with $20 \mu \mathrm{g} / \mathrm{mL}$ AF594 under vigorous agitation. Stained bacteria were washed twice with PBS and used for i.v. infections.

Mouse infections and in vivo treatments. For infection experiments, $S$. aureus strains were subcultured for 2 hours without antibiotics until the exponential phase (OD $660 \mathrm{~nm}, 1.0$ ), washed with saline once, and then resuspended in saline and injected i.v. into the tail vein or i.p. at $5 \times 10^{7} \mathrm{CFU}$ in $200 \mu \mathrm{L}$ saline. Mice were monitored and sacrificed at various time points according to the experimental design.

Fifty $\mathrm{mg} / \mathrm{kg}$ vancomycin (Sigma-Aldrich) or liposome-encapsulated vancomycin (vancosomes; ref. 44) was administered either 24 hours, 24, and 48 hours, or 24,48 , and 72 hours after infection either i.v. or i.p. In combined treatments, mice received $25 \mathrm{mg} / \mathrm{kg}$ vancomycin i.v and 25 $\mathrm{mg} / \mathrm{kg}$ vancosomes or vancomycin i.p. CFU were determined after 72 hours and survival was monitored closely until 10 days after infection. Mice that lost more than $20 \%$ of their body weight were euthanized. For Cytospins and flow cytometry, 6-mg vancosomes were labeled with $5 \mu \mathrm{L}$ DID solution (Thermo Fisher Scientific) in $1 \mathrm{~mL}$ saline for 30 minutes and washed twice before injection.

Bacteriological analysis. Anesthetized mice were washed with 70\% ethanol under aseptic conditions. Blood was collected in a heparinized syringe by cardiac puncture. This step did not result in full perfusion of organs; however, in comparing perfused organs with those that were not perfused, no differences in solid organs were seen (data not shown). Peritoneal lavage was performed with $7 \mathrm{~mL}$ sterile PBS. The lungs, liver, kidneys, spleen, peritoneal wall, and visceral associated fat were removed after thoracotomy, weighed, and homogenized. For determination of CFU, $30 \mu \mathrm{L}$ tissue homogenate, blood, or lavage was serially diluted, plated onto brain-heart infusion agar plates, and incubated at $37^{\circ} \mathrm{C}$ for 18 hours, and then bacterial colonies were counted.

Ex vivo time-lapse microscopy. Kupffer cells were isolated as described previously (45). Briefly, the abdominal cavity of anesthetized mice was opened to cannulate the inferior vena cava. The liver was perfused in situ first with $\mathrm{Ca}^{2+}$-, $\mathrm{Mg}^{2+}$-free HBSS, and then with HBSS containing 0.05\% collagenase type IV (Worthington Biochemical Corp.), $0.025 \%$ pronase E (US Biological), and 0.02\% DNase I (Roche Diagnostics) at a flow rate of $4 \mathrm{~mL} / \mathrm{min}$. After perfusion, tissue was further digested in HBSS containing $0.009 \%$ collagenase type IV, $0.009 \%$ pronase $\mathrm{E}$, and $0.02 \%$ DNase I at $37^{\circ} \mathrm{C}$ for 30 minutes. The cell suspension was passed through a $100-\mu \mathrm{m}$ nylon filter and centrifuged at $25 \mathrm{~g}$ for 5 minutes at room temperature to remove the hepatocytes. The supernatant was transferred to a new tube and centrifuged at $400 \mathrm{~g}$ for 10 minutes at $4^{\circ} \mathrm{C}$. Subsequently, the cell pellets were resuspended in $17 \%$ iodixanol (Axis-Shield PoC) solution and centrifuged at $400 \mathrm{~g}$ for 15 minutes at $20^{\circ} \mathrm{C}$. From this nonparenchymal fraction, Kupffer cells were positively selected with anti-F4/80 microbeads and MidiMACS column (Miltenyi Biotec).

Alternatively, liver was removed from 0.5-hour infected mice, cauterized to close vessels, and incubated in DMEM with 10\% FCS for microcopy in the IncuCyte ZOOM system (Essen BioScience) as described in the information to follow.

For isolation of peritoneal macrophages or neutrophils, peritoneal lavage was performed with $7 \mathrm{~mL}$ sterile PBS, and cells were centrifuged at $400 \mathrm{~g}$ prior to positive magnetic-activated cell sorting (MACS) separation with either anti-F4/80 microbeads or PE-Ly6G (clone 1A8) and anti-PE microbeads.

Cells were cultured in 96-well plates in DMEM with 10\% FCS and for time-lapse microscopy placed in the IncuCyte ZOOM system. The microscope was equipped for phase contrast and 2 fluorescent channels (green excitation 440-480 nm [Ex440-480 nm], emission 504-544 nm [Em504-544 nm], and red Ex565-605 nm, Em625-705 nm) and with the Basler scout scA1400-30gm monochrome camera (resolution $1392 \times 1040$ pixels; pixel size $6.45 \times 6.45 \mu \mathrm{m}$; frame rate, 30 frames per second). Cells were imaged with a $\times 20$ air objective (NA 0.40). Cell counting was performed using Image J software version 1.52A. Eight-bit grayscale images from the start of imaging were exported from IncuCyte ZOOM version 2018A. Images were smoothed and adjusted using the threshold function in ImageJ. The fill holes and watershed functions were applied to separate clumped cells. Finally, cell counting was performed through a particle analysis excluding noise particles smaller than 45 pixels or those with a circularity lower than 0.4. Infected cell counting was performed using the IncuCyte ZOOM version 2018A basic analyzer software. A top-hat transform with a radius of $10.0 \mu \mathrm{m}$ was used to calculate a background-subtracted image before green particles were analyzed with a threshold of 1.50 green calibrated unites (GCU), edge split off, with a hole fill of $5.0 \mu \mathrm{m}^{2}$. The number of cells that were overgrown was counted manually. Four fields of view (FOVs) were analyzed per mouse and counted as $n=1$.

IVM. A multichannel 2-photon IVM was used to image mouse kidneys. A spinning-disk IVM was used to image the mouse livers and peritoneal cavities. Imaging of the kidney was performed as described previously $(46,47)$. In brief, mice were anesthetized with ketamine $(200$ $\mathrm{mg} / \mathrm{kg}$ ) and xylazine $(10 \mathrm{mg} / \mathrm{kg})$. The right jugular vein was cannulated to enable administration of antibodies and additional anesthetic. Mice 
were shaved to remove the fur from the upper left flank region, incisions in the skin and abdominal wall were performed, and the left kidney was exteriorized without removing the renal capsule or interrupting blood flow. For upright imaging, mice were placed on a heating pad, and the kidney was immobilized in a heated well incorporated into a custombuilt stage. The kidney was stabilized with vacuum grease, bathed in normal saline, and covered with a coverslip. For inverted imaging, mice were positioned with the exteriorized kidney facing down on a heating plate incorporating a coverslip, and the kidney was bathed in saline. To identify neutrophils, PE-conjugated anti-mouse Ly6G (clone 1A8, BioLegend, $2 \mu \mathrm{g})$ was used, and either Q-tracker $655(2.5 \mu \mathrm{L})$ or AF680albumin $(30 \mu \mathrm{L})$ was used to label the vasculature, in each case injected i.v. The renal microvasculature was observed with an upright Leica SP8 2-photon confocal microscope (Leica Microsystems), equipped with a $25 \times 0.95$ NA water objective lens, InSight DeepSee pulsed infrared lasers with dual output (fixed $1040 \mathrm{~nm}$ and tunable 680-1300 nm; Spectra-Physics), and a high-speed 8-kHz resonant scanner. Emitted fluorescence was detected by non-descanned HyD detectors with 650-700 nm, 565-620 nm, and 500-550 nm. Alternatively, an inverted Leica SP8 DIVE multiphoton microscope equipped with a 40x 1.1 NA water objective lens, an InSight X3 pulsed infrared laser with dual output (fixed $1045 \mathrm{~nm}$ and tunable 680-1300 nm; Spectra-Physics), a high-speed $8-\mathrm{kHz}$ tandem scanner, and a 4 Tune non-descanned detector with 4-channel hybrid detectors was used.

Imaging of the liver was performed as described previously (48). Kupffer cells were labeled with i.v. injection of an APC 780-conjugated anti-mouse F4/80 antibody (clone BM8, eBioscience; $5 \mu \mathrm{L}$ ). The liver surface was labelled directly before imaging by superfusion in vivo (24) with an eF660-conjugated anti-mouse-podoplanin antibody (clone eBio8.1.1, eBioscience, $2.5 \mu \mathrm{L}$ in $250 \mu \mathrm{L}$ PBS) to label the mesothelium. Hepatocytes become only visible (autofluorescence) when mesothelium was ruptured. Image acquisition for liver was performed using a spinning-disc confocal Olympus IX81 inverted microscope, equipped with an Olympus focus drive and a motorized stage (Applied Scientific Instrumentation, Eugene, OR, USA) and fitted with a motorized objective turret equipped with $4 \times / 0.16$ UPLANSAPO, $10 \times / 0.40$ UPLANSAPO, and 20 $\times / 0.70$ UPLANSAPO objective lenses and coupled to a confocal light path (WaveFx; Quorum Technologies) based on a modified Yokogawa CSU-10 head (Yokogawa Electric Corporation).

Assessment of renal injury. Serum creatinine levels were determined with a QuantiChrom Creatinine Assay Kit (DICT-500, BioAssay Systems). The number of dead cells in kidney tubules was assessed with a 2-photon IVM 5-10 minutes after i.v. injection of Sytox Orange ( $5 \mu \mathrm{M}$, Thermo Fisher Scientific).

Flow cytometry. Single-cell suspensions from peritoneal lavage were collected as described (17). The gating strategy to separate LPMs, SPMs, monocytes, and neutrophils is shown in Supplemental Figure 3. Kidneys were dissected, and fat and connective tissue carefully removed before digestion in Collagenase/DNase $(1 \mathrm{mg} / \mathrm{mL} / 100 \mu \mathrm{g} /$ $\mathrm{mL}$, Sigma-Aldrich) in RPMI with $10 \%$ FCS for 45 minutes. Tubular fragments from digested kidneys were removed by filtration through a 70- $\mu \mathrm{m}$ nylon filter. After erythrocyte lyses with ammoniumchloride-potassium (ACK) lysing buffer (Gibco, Thermo Fisher Scientiric) and Fc-blocking (anti-CD16/32 antibody, clone 2.4G2, Bio X Cell), cells from kidney, peritoneal lavage or blood were stained for 30 minutes with specific markers including BV510-conjugated antimouse CD45 (clone 30-F11, BioLegend, 1:200), PE-Cy7-conjugated anti-mouse CD11b (clone M1/70, eBioscience, 1:1000), eF450conjugated anti-mouse F4/80 (clone BM8, eBioscience, 1:100), PerCP-Cy5.5-conjugated anti-mouse Ly6C (clone HK1.4, BioLegend, 1:100), APC-Cy7-conjugated anti-mouse Ly6G (clone 1A8, BioLegend, 1:100), APC-conjugated anti-mouse Ly6G (clone 1A8, BioLegend, 1:100), eF660-conjugated anti-mouse CD11c (clone N418, eBioscience, 1:100), and PE-conjugated anti-mouse MHCII (clone M5/114.15.2, Pharmingen, 1:100). GATA6 was detected using rabbit anti-GATA6 (D61E4, Cell Signaling Technology) and AF647-conjugated anti-rabbit IgG secondary antibody (Life Technologies, Thermo Fisher Scientific). Appropriate isotype control antibodies were used to confirm positive signals. Nonviable cells were identified with Ghost viability dye-AF700 (Tonbo Biosciences, 1:1000) or viability dye eF780 (eBioscience, 1:1000). For intracellular staining, the Foxp3 staining kit (Thermo Fisher Scientific) was used.

Samples were run using a flow cytometer (FACSCanto, BD) and analyzed using FlowJo software (Tree Star). Total cell numbers were calculated by using 123 count eBeads Counting Beads (Thermo Fisher Scientific) according to the manufacturer's instructions. Bacteria were declared as bound or ingested when the cell population of interest had a $\mathrm{GFP}^{+}$population not detected in uninfected mice. The numbers of free bacteria were determined in samples by including smaller (FSC 200/ SSC 200) GFP' particles and excluding cells (CD45 ) in analysis. For blood neutrophil counts, flow cytometry analysis was carried out using the Attune Flow Cytometer (Life Technologies, Thermo Fisher Scientific). The Attune Flow Cytometer collects event data from a specific volume and as such provides an exact event number used to calculate the exact number of cells in a sample of known volume.

Cytospin. Cell harvesting of peritoneal cavity and Fc blocking was identical to flow cytometry. One hundred microliters of cell suspension $\left(1 \times 10^{6}\right.$ cells $\left./ \mathrm{mL}\right)$ was transferred into the chambers of the Cytospin. Samples were run at $200 \mathrm{~g}$ for 6 minutes and after drying, the samples were stained with AF750-conjugated anti-mouse F4/80 (clone BM8, AbLab, 1:200) and eFluor 660-conjugated anti-mouse CD11b (clone M1/70, eBiosicene, 1:200) antibodies for 30 minutes and fixed with $1 \%$ paraformaldehyde.

ELISA. Peritoneal lavage of mice was performed, samples were centrifuged, and supernatant was used to determine CXCL1 and CXCL2 via ELISA (R\&D Systems, Mouse Quantikine ELISA Kit).

Statistics. Statistical analysis was performed with GraphPad Prism 7 software (GraphPad Software). Two groups were compared by using an unpaired 2-tailed Student's $t$ test. Means among 3 or more groups were compared with 1-way or 2-way ANOVA followed by Bonferroni's post hoc test for multiple comparisons adjustment. Bacterial CFU data were compared by Kruskal-Wallis test followed by Dunn's multiple comparisons test. Survival curves were compared using log-rank (MantelCox) test. A $P$ value of less than 0.05 was considered significant.

Study approval. All experimental procedures were approved by the University of Calgary Animal Care Committee and were in compliance with the Canadian Council for Animal Care Guidelines.

\section{Author contributions}

SKJ, MJH, and PK conceived the project and designed the experiments. SKJ, BGJS, MH, MP, CD, JD, AB, and FvdW performed the experiments and analyzed the data. AO prepared and characterized vancosomes. All authors discussed the results, and SKJ, MJH, and PK wrote the manuscript. 


\section{Acknowledgments}

We thank Trecia Nussbaumer from the Snyder Institute for Chronic Diseases for the breeding of mice, Karen Poon from the Snyder Institute Molecular Core for assistance with flow cytometry, and the University of Calgary Live Cell Imaging facility for assistance with microscopy techniques. We also thank Janos Peti-Peterdi from the University of Southern California for teaching us how to use the Leica-Dive 2-photon microscope to visualize glomeruli. SKJ (JO 1497/2-1), MP (PE 2737/1-1), and CD (DE 2654/1-1) are supported by Deutsche Forschungsgemeinschaft (DFG) Research Fellowships; BGJS is supported by a postdoctoral fellowship from CIHR; and $\mathrm{MH}$ has a postdoctoral fellowship from the Alberta Cancer Foundation. MJH is a National Health and Medical Research Council (Australia) Senior Research Fellow (grant ID 1042775). PK is funded by a CIHR Foundation Grant.

Address correspondence to: Paul Kubes, HRIC 4AA16, University of Calgary, 3330 Hospital Drive Northwest, Calgary, Alberta, T2N 4N1, Canada. Phone: 403.220.2705; Email: pkubes@ucalgary.ca. Or to: Michael J. Hickey, Level 5, Block E, Monash Medical Centre, 246 Clayton Road, 3168 Clayton, Victoria, Australia. Phone: 61.3.8572.2591; Email: michael.hickey@monash.edu.
1. Thwaites GE, et al. Clinical management of Staphylococcus aureus bacteraemia. Lancet Infect Dis. 2011;11(3):208-222.

2. Pottinger PS. Methicillin-resistant Staphylococcus aureus infections. Med Clin North Am. 2013;97(4):601-619.

3. Saginur R, Suh KN. Staphylococcus aureus bacteraemia of unknown primary source: where do we stand? Int J Antimicrob Agents. 2008; (32 suppl 1):S21-S25.

4. Lehar SM, et al. Novel antibody-antibiotic conjugate eliminates intracellular $S$. aureus. Nature. 2015;527(7578):323-328.

5. Kullar R, Davis SL, Levine DP, Rybak MJ. Impact of vancomycin exposure on outcomes in patients with methicillin-resistant Staphylococcus aureus bacteremia: support for consensus guidelines suggested targets. Clin Infect Dis. 2011;52(8):975-981.

6. Rogers DE. Studies on bacteriemia. I. Mechanisms relating to the persistence of bacteriemia in rabbits following the intravenous injection of staphylococci. J Exp Med.1956;103(6):713-742.

7. Thwaites GE, Gant V. Are bloodstream leukocytes Trojan Horses for the metastasis of Staphylococcus aureus? Nat Rev Microbiol. 2011;9(3):215-222.

8. Gresham HD, Lowrance JH, Caver TE, Wilson BS, Cheung AL, Lindberg FP. Survival of Staphylococcus aureus inside neutrophils contributes to infection. JImmunol. 2000;164(7):3713-3722.

9. Flannagan RS, Heit B, Heinrichs DE. Antimicrobial mechanisms of macrophages and the immune evasion strategies of Staphylococcus aureus. Pathogens. 2015;4(4):826-868.

10. Surewaard BG, et al. Identification and treatment of the Staphylococcus aureus reservoir in vivo. JExp Med. 2016;213(7):1141-1151.

11. Rollin G, et al. Intracellular survival of Staphylococcus aureus in endothelial cells: a matter of growth or persistence. Front Microbiol. 2017;8:1354.

12. Fraunholz M, Sinha B. Intracellular Staphylococcus aureus: live-in and let die. Front Cell Infect Microbiol. 2012;2:43.

13. Altman DR, et al. Transmission of methicillinresistant Staphylococcus aureus via deceased donor liver transplantation confirmed by whole genome sequencing. Am J Transplant. 2014;14(11):2640-2644.

14. Zeng Z, Surewaard BG, Wong CH, Geoghegan JA, Jenne CN, Kubes P. CRIg functions as a macrophage pattern recognition receptor to directly bind and capture blood-borne gram-positive bacteria. Cell Host Microbe. 2016;20(1):99-106.
15. Patel N, Huang D, Lodise T. Potential for cost saving with iclaprim owing to avoidance of vancomycin-associated acute kidney injury in hospitalized patients with acute bacterial skin and skin structure infections. Clin Drug Investig. 2018;38(10):935-943.

16. Rauch S, DeDent AC, Kim HK, Bubeck Wardenburg J, Missiakas DM, Schneewind O. Abscess formation and $\alpha$-hemolysin induced toxicity in a mouse model of Staphylococcus aureus peritoneal infection. Infect Immun. 2012;80(10):3721-3732.

17. Ghosn EE, et al. Two physically, functionally, and developmentally distinct peritoneal macrophage subsets. Proc Natl Acad Sci U S A. 2010;107(6):2568-2573.

18. Okabe Y, Medzhitov R. Tissue-specific signals control reversible program of localization and functional polarization of macrophages. Cell. 2014;157(4):832-844

19. Barth MW, Hendrzak JA, Melnicoff MJ, Morahan PS. Review of the macrophage disappearance reaction. J Leukoc Biol. 1995;57(3):361-367.

20. Mullaly SC, Kubes P. The role of TLR 2 in vivo following challenge with Staphylococcus aureus and prototypic ligands. J Immunol. 2006;177(11):8154-8163.

21. Crosara-Alberto DP, Darini AL, Inoue RY, Silva JS, Ferreira SH, Cunha FQ. Involvement of NO in the failure of neutrophil migration in sepsis induced by Staphylococcus aureus. Br J Pharmacol. 2002;136(5):645-658.

22. Leech JM, Lacey KA, Mulcahy ME, Medina E, McLoughlin RM. IL-10 plays opposing roles during Staphylococcus aureus systemic and localized infections. JImmunol. 2017;198(6):2352-2365.

23. Rosas M, et al. The transcription factor Gata6 links tissue macrophage phenotype and proliferative renewal. Science. 2014;344(6184):645-648

24. Wang J, Kubes P. A reservoir of mature cavity macrophages that can rapidly invade visceral organs to affect tissue repair. Cell. 2016;165(3):668-678.

25. Cheng AG, Kim HK, Burts ML, Krausz T, Schneewind O, Missiakas DM. Genetic requirements for Staphylococcus aureus abscess formation and persistence in host tissues. FASEB J. 2009;23(10):3393-3404.

26. Stamatiades EG, et al. Immune monitoring of trans-endothelial transport by kidney-resident macrophages. Cell. 2016;166(4):991-1003.

27. Yatim KM, Gosto M, Humar R, Williams AL, Oberbarnscheidt MH. Renal dendritic cells sample blood-borne antigen and guide T-cell migration to the kidney by means of intravascular processes. Kidney Int. 2016;90(4):818-827.

28. Surewaard BGJ, et al. $\alpha$-toxin induces platelet aggregation and liver injury during Staphylococcus aureus sepsis. Cell Host Microbe. 2018;24(2):271-284.e3.

29. Bouma G, Ancliff PJ, Thrasher AJ, Burns SO. Recent advances in the understanding of genetic defects of neutrophil number and function. $\mathrm{Br} \mathrm{J}$ Haematol. 2010;151(4):312-326.

30. Bodey GP, Buckley M, Sathe YS, Freireich EJ. Quantitative relationships between circulating leukocytes and infection in patients with acute leukemia. Ann Intern Med. 1966;64(2):328-340.

31. Gregory SH, Sagnimeni AJ, Wing EJ. Bacteria in the bloodstream are trapped in the liver and killed by immigrating neutrophils. J Immunol. 1996;157(6):2514-2520.

32. Voyich JM, et al. Insights into mechanisms used by Staphylococcus aureus to avoid destruction by human neutrophils. J Immunol. 2005;175(6):3907-3919.

33. Ferraioli G, et al. Percutaneous and surgical treatment of pyogenic liver abscesses: observation over a 21-year period in 148 patients. Dig Liver Dis. 2008;40(8):690-696.

34. Jun $\mathrm{CH}$, et al. Risk factors and clinical outcomes for spontaneous rupture of pyogenic liver abscess. J Dig Dis. 2015;16(1):31-36.

35. Cassado Ados A, D'Império Lima MR, Bortoluci KR. Revisiting mouse peritoneal macrophages: heterogeneity, development, and function. Front Immunol. 2015;6:225.

36. Cassado Ados A, et al. Cellular renewal and improvement of local cell effector activity in peritoneal cavity in response to infectious stimuli. PLoS One. 2011;6(7):e22141.

37. Abtin A, et al. Perivascular macrophages mediate neutrophil recruitment during bacterial skin infection. Nat Immunol. 2014;15(1):45-53.

38. Yao Y, et al. Induction of autonomous memory alveolar macrophages requires $\mathrm{T}$ cell help and is critical to trained immunity. Cell. 2018;175(6):1634-1650.e17

39. De Filippo K, et al. Mast cell and macrophage chemokines CXCL1/CXCL2 control the early stage of neutrophil recruitment during tissue inflammation. Blood. 2013;121(24):4930-4937.

40. Kim ND, Luster AD. The role of tissue resident cells in neutrophil recruitment. Trends Immunol. 2015;36(9):547-555.

41. Tuchscherr L, et al. Staphylococcus aureus phenotype switching: an effective bacterial 
strategy to escape host immune response and establish a chronic infection. EMBO Mol Med. 2011;3(3):129-141.

42. A-Gonzalez N, et al. Phagocytosis imprints heterogeneity in tissue-resident macrophages. JExp Med. 2017;214(5):1281-1296.

43. Horn J, Stelzner K, Rudel T, Fraunholz M. Inside job: Staphylococcus aureus host-pathogen interactions. Int J Med Microbiol. 2018;308(6):607-624.
44. Sande L, et al. Liposomal encapsulation of vancomycin improves killing of methicillin-resistant Staphylococcus aureus in a murine infection model. JAntimicrob Chemother. 2012;67(9):2191-2194.

45. Lee WY, et al. An intravascular immune response to Borrelia burgdorferi involves Kupffer cells and iNKT cells. Nat Immunol. 2010;11(4):295-302.

46. Devi S, et al. Multiphoton imaging reveals a new leukocyte recruitment paradigm in the glomeru- lus. Nat Med. 2013;19(1):107-112

47. Snelgrove SL, et al. Activated renal dendritic cells cross present intrarenal antigens after ischemia-reperfusion injury. Transplantation . 2017;101(5):1013-1024.

48. Wong CH, Jenne CN, Lee WY, Léger C, Kubes P. Functional innervation of hepatic iNKT cells is immunosuppressive following stroke. Science. 2011;334(6052):101-105. 